\title{
‘Measurement of Quantum Mechanical Operators’ Revisited
}

\author{
Leon Loveridge ${ }^{\mathrm{a}}$ and Paul Busch ${ }^{\mathrm{b}}$ \\ University of York, Department of Mathematics, Mathematical Physics Section, York, UK
}

To appear in: Eur. Phys. J. D (2011)

\begin{abstract}
The Wigner-Araki-Yanase (WAY) theorem states a remarkable limitation to quantum mechanical measurements in the presence of additive conserved quantities. Discovered by Wigner in 1952, this limitation is known to induce constraints on the control of individual quantum systems in the context of information processing. It is therefore important to understand the precise conditions and scope of the WAY theorem. Here we elucidate its crucial assumptions, briefly review some generalizations, and show how a particular extension can be obtained by a simple modification of the original proofs. We also describe the evolution of the WAY theorem from a strict no-go verdict for certain, highly idealized, precise measurements into a quantitative constraint on the accuracy and approximate repeatability of imprecise measurements.
\end{abstract}

PACS. 03.65.Ta Foundations of quantum mechanics; measurement theory - 03.67.-a Quantum information

\section{Introduction}

Quantum mechanical experiments involving the manipulation of individual quantum objects no longer reside only in the minds of a few theoretical physicists, but are a routine occurrence across many physical disciplines such as quantum optics and quantum information. This not only provides new and exciting opportunities for future technologies such as quantum computing, but necessitates a fundamental re-examination of the quantum mechanical formalism itself, and a new understanding of its role in modern applications. With the ever-decreasing size of the components involved in these technologies, it is both interesting from a foundational viewpoint and important in more practical respects to understand any fundamental limitations on the possible size of such microscopic instruments.

One such limitation arises as a consequence of conservation laws for additive quantities that do not commute with the observable to be measured. Whilst considering spin- $-\frac{1}{2}$ measurements, Wigner [1] discovered that the total angular momentum of the object plus apparatus cannot be conserved in an accurate and repeatable measurement of a particular component. This observation was soon stated in greater generality as a theorem by Araki and Yanase 2 that has become known as the Wigner-Araki-Yanase (WAY) theorem. Despite the fact that the original papers [1] and 2] have been widely noted and the WAY theorem has been extended in various respects, its full scope is still unknown.

\footnotetext{
a e-mail: ldl500@york.ac.uk

b e-mail: paul.busch@york.ac.uk
}

It is the purpose of this paper to survey the evolution of formulations of WAY-type theorems, elucidate the significance of the underlying assumptions, and clarify the general structure and extent of such theorems. We will also provide some new extensions of known results and propose an answer to a long-standing question concerning the possibility of momentum-conserving measurements of the position of a quantum particle.

In Sec. 3] we revisit Wigner's 1952 paper [1]. In particular we scrutinize the final page where Wigner examines the consequences of dropping the assumption that the measurement be repeatable. This is a relaxation which is physically relevant, but is still not appreciated by many practitioners of quantum theory. Wigner notes that in this case the issue arises of the distinguishability of the states of the measuring apparatus, given that the limitation imposed by the conservation law also applies to a measurement of the pointer. The paper 1 is written (in German) with the simplicity and elegance characteristic of Wigner; in order to make it more widely accessible, a translation into English is provided as a concurrent publication [3].

In Sec. 4 we proceed to give a modification of the proof of Araki and Yanase 22 leading to a sharpening and extension of the WAY theorem. They prove for certain classes of observables and conserved quantities that under the assumption of accuracy and repeatability, the observable to be measured must commute with the (object part of) the conserved quantity. Here we show that the same conclusion follows if the repeatability of the measurement is replaced by the assumption that the pointer observable commutes with the conserved quantity. This condition, which following Ozawa 4 we shall call Yanase condition, 
was already alluded to in 1 and 5 . In fact, the WAY theorem also precludes accurate and repeatable measurements of the pointer observable (given the conservation law) unless the Yanase condition is fulfilled.

In Sec. 5 we review formulations of WAY-type limitations for approximate measurements. In particular we present and develop an inequality first formulated by Ozawa [4 that demonstrates trade-off relations between a measure of error and the "size" of the apparatus (suitably defined). In Sec. 6] we revisit some model measurement schemes, notably by Ohira and Pearle [6, and observe that the "ways out" of the WAY limitation sought there always come at the expense of violating the repeatability and Yanase conditions. This helps to highlight the fact that the WAY theorem is often paraphrased in a superficial way, ignoring the repeatability property and the relevance of the Yanase condition.

Sec. 7 contains a description of the largely unexplored question of whether position measurements that respect momentum conservation are subject to a WAY-type limitation. Here we adapt Ozawa's inequality to establish the necessity of a large apparatus for good measurements, provided that the Yanase condition is satisfied. We also formulate a trade-off inequality analogous to Ozawa's inequality with which one can quantify the degree of repeatability achievable given the size of the apparatus. Finally we provide an affirmative answer, in a certain approximate sense, to a problem posed by Stein and Shimony in 1979 [7] concerning the feasibility of repeatable position measurements obeying momentum conservation.

The paper concludes with some remarks on the relavance of the WAY theorem in contemporary quantum physics and quantum information.

We begin with an outline of concepts of quantum measurement theory relevant to our investigation.

\section{Preliminaries}

We will apply the standard formulation of quantum measurements (e.g., 8]) where the quantum system and apparatus are represented by complex Hilbert spaces $\mathcal{H}$ and $\mathcal{K}$, respectively. These come equipped with the usual inner products denoted $\langle\cdot \mid \cdot\rangle$. Observables are given as positive operator valued measures (POVMs) $\mathrm{E}: X \mapsto \mathrm{E}(X)$. The operators $\mathrm{E}(X)$ associated with subsets $X$ of $\mathbb{R}$ are positive operators whose expectation values $\langle\psi \mid \mathrm{E}(X) \psi\rangle$ (for normalized states $\psi \in \mathcal{H}$ ) represent the measurement outcome probabilities of finding a result in $X$; these operators are called effects. If these effects are projections, the POVM E is a spectral measure from which one recovers the standard representation of an observable as a self-adjoint operator, namely $\int x \mathrm{E}(d x) \equiv M$.

For a measurement to take place, there must be an interaction between the object system and a macroscopic measuring apparatus, whereby the experimenter can read off the values of the measured observable. The part of this device that actually comes into contact with the quantum system under investigation may only be a small component of the whole apparatus (and could be referred to as a probe). We shall not discuss the process of amplification by which information from the interaction generates macroscopic outcome values.

The composite system-apparatus Hilbert space is described by the tensor product $\mathcal{H}_{T}:=\mathcal{H} \otimes \mathcal{K}$. The time evolution of the system-plus-apparatus is then given by a unitary operator $U$ on $\mathcal{H}_{T}$, which serves to couple the states of the system to those of the apparatus during an interaction period $\tau$. In order that this interaction may be said to lead to a measurement of an observable E, some extra elements are still required; these are a self-adjoint operator $Z$ on $\mathcal{K}$, which represents a "pointer observable", and a (fixed) initial apparatus state in $\mathcal{K}$, chosen to be the pure (vector) state $\phi$, with $\|\phi\|=1$.

We then define a measurement $\mathcal{M}$ of an observable $\mathrm{E}$ as the 5 -tuple $\langle\mathcal{K}, U, \phi, Z, f\rangle$ satisfying the probability reproducibility condition; that the outcome distribution for $\mathrm{E}$ in any state $\varphi$ be recovered from the pointer statistics in the final state $\Psi_{\tau}=U(\varphi \otimes \phi) \in \mathcal{H}_{T}$. This condition can be written symbolically as

$$
\left\langle\Psi_{\tau} \mid \mathbf{1} \otimes \mathrm{E}^{Z}\left(f^{-1}(X)\right) \Psi_{\tau}\right\rangle \equiv\langle\varphi \mid \mathrm{E}(X) \varphi\rangle,
$$

where $\mathrm{E}^{Z}\left(f^{-1}(X)\right)$ are spectral projections of $Z$, and (1) holds for all $\varphi$ and $X$. Conversely, given a measurement scheme as described above, this relation determines the measured observable $\mathrm{E}$. The scaling function $f$ is used to map the values of the pointer to those of the measured observable.

A measurement is said to be repeatable if, upon immediate repetition of the measurement, the same outcome is achieved with certainty. This may be written:

$$
\left\langle\Psi_{\tau} \mid \mathrm{E}(X) \otimes \mathrm{E}^{Z}\left(f^{-1}(X)\right) \Psi_{\tau}\right\rangle=\langle\varphi \mid \mathrm{E}(X) \varphi\rangle .
$$

It should be noted that even when (11) holds, it is not guaranteed that (2) is satisfied, and as such the question of repeatability must be treated independently of that of probability reproducibility. Conditions. (11) and (2) can be rephrased in a more concise form as follows: if the outcome of the first measurement is described by a set $X$, the system's state is obtained by taking a partial trace operation:

$$
\rho_{X}=\operatorname{tr}_{\mathcal{K}}\left[\mathbf{1} \otimes \mathrm{E}^{Z}\left(f^{-1}(X)\right)\left|\Psi_{\tau}\right\rangle\left\langle\Psi_{\tau}\right|\right] ;
$$

Now condition (1) reads:

$$
\operatorname{tr}\left[\rho_{X}\right]=\langle\varphi \mid \mathrm{E}(X) \varphi\rangle .
$$

Writing $\hat{\rho}_{X}=\rho_{X} / \operatorname{tr}\left[\rho_{X}\right]$ for the normalized state, the repeatability condition (2) then becomes:

$$
\operatorname{tr}\left[\hat{\rho}_{X} \mathrm{E}(X)\right]=1 \text {. }
$$

Although in many textbooks the term measurement is understood as comprising the repeatability property, it is important to recognize that most realistic measurements are not repeatable. Furthermore, measurements are rarely accurate and the actually measured observable is appropriately described as a POVM. We will see below that even as early as 1952 Wigner was working with more general measurement models that do not satisfy the repeatability criterion and whose associated observable is a POVM. 


\section{Wigner 1952}

\subsection{Wigner's example}

Wigner first noticed that repeatable measurements of the $x$-component of the spin of a spin- $\frac{1}{2}$ particle necessarily violate the conservation of the $z$-component of the total angular momentum of the system plus apparatus, written $S_{z} \otimes \mathbf{1}+\mathbf{1} \otimes J_{z}$. He also demonstrated the feasibility of recovering arbitrarily accurate and repeatable measurements if the apparatus becomes "large". This is a significant feature in much of the work following Wigner's discovery, and we sketch the argument here. We continue with the notation that $\phi \in \mathcal{K}$ represents the initial (normalized) apparatus state, and $\phi_{ \pm} \in \mathcal{K}$ orthonormal pointer states, and throughout we shall choose units where $\hbar=1$. The unitary evolution takes the form (with $\varphi_{ \pm}$representing $S_{x}$ eigenstates):

$$
\begin{aligned}
& \varphi_{+} \otimes \phi \longrightarrow \varphi_{+} \otimes \phi_{+}, \\
& \varphi_{-} \otimes \phi \longrightarrow \varphi_{-} \otimes \phi_{-} ;
\end{aligned}
$$

the evolution for the eigenstates $\psi_{ \pm}=\left(\varphi_{+} \pm \varphi_{-}\right) / \sqrt{2}$ of $S_{z}$ is then

$$
\begin{aligned}
& \psi_{+} \otimes \phi \longrightarrow \frac{1}{2}\left[\psi_{+} \otimes\left(\phi_{+}+\phi_{-}\right)+\psi_{-} \otimes\left(\phi_{+}-\phi_{-}\right)\right], \\
& \psi_{-} \otimes \phi \longrightarrow \frac{1}{2}\left[\psi_{+} \otimes\left(\phi_{+}-\phi_{-}\right)+\psi_{-} \otimes\left(\phi_{+}+\phi_{-}\right)\right] .
\end{aligned}
$$

This violates angular momentum conservation, since the expectations $\left\langle S_{z}+J_{z}\right\rangle$ agree on the right hand sides of (8) and (9) but differ by one unit on the left hand sides. Since, as Wigner argues, spin component measurements are "practically possible", he introduces the following modification in order to model an approximate realization of the measurement:

$$
\begin{aligned}
& \varphi_{+} \otimes \phi \longrightarrow \varphi_{+} \otimes \phi_{+}+\varphi_{-} \otimes \eta, \\
& \varphi_{-} \otimes \phi \longrightarrow \varphi_{-} \otimes \phi_{-}-\varphi_{+} \otimes \eta,
\end{aligned}
$$

with $\left\langle\eta, \phi_{ \pm}\right\rangle=0$. There are now three (un-normalized) pointer states, representing a three-outcome measurement, the third (labelled by $\eta$ ) corresponding to an undetermined spin, representing a situation where the apparatus cannot identify a definite spin. The two definite outcomes are represented by effects $E_{ \pm}=\left(1-\|\eta\|^{2}\right) P\left[\varphi_{ \pm}\right]$, and the third is represented by a trivial effect $E_{0}=\|\eta\|^{2} \mathbf{1}$ (with probability given by $\|\eta\|^{2}$ ). Wigner shows that $\|\eta\|^{2}$ can be made arbitrarily small given a "large" apparatus. Specifically he shows that if the state $\phi$ has a very large number of components in its expansion in terms of $J_{z^{-}}$ eigenvectors $\phi_{\nu}$, so that $\phi=\sum_{\nu=1}^{n} \phi_{\nu}$, then with some suitable assumptions and conditions, $\|\eta\|^{2}=1 /(2 n-1)$. Thus in the large- $n$ limit, $\|\eta\| \rightarrow 0$ and accurate and repeatable measurements are, to a very good approximation, recovered.

We note that the large size of the apparatus is used here only as a sufficient condition to achieve good measurement accuracy; the argument does not yield it as a necessary one.

\subsection{Implications of dropping repeatability}

Wigner's consideration in his final page is intriguing although very sketchy and somewhat open-ended; there he discusses a more general measurement scheme in which the repeatability restriction is dropped. We carefully reconstruct his argument in the Appendix; here we provide a more concise and more general calculation, which contains Wigner's conclusion as a special case. This approach has considerably less cumbersome algebra, and relies on exploiting the condition that the interaction must be a measurement (in the sense of (1)) from the beginning. We make no assumption on the product form of the final states, and allow the most general (entangled) final state in the system-apparatus Hilbert space.

For notational convenience and following Wigner, when required we shift the spectral values of the observables concerned in order that they are integers; for example the eigenvalues of the object part of the conserved quantity are now 0 and 1 . In contrast to Wigner, we do not make the assumption that the spectrum of the apparatus' conserved quantity is bounded below by zero. With $\chi_{k}^{\prime}, \chi_{k}^{\prime \prime}, \phi_{k}^{\prime}$ and $\phi_{k}^{\prime \prime}$ representing (un-normalized) eigenstates of $J_{z}$ and $\psi_{0}, \psi_{1}$ (normalized) $S_{z}$ eigenstates, the unitary evolution $U$ gives:

$$
\begin{aligned}
& \left(\psi_{0}+\psi_{1}\right) \otimes \sum \phi_{k} \stackrel{U}{\longrightarrow} \psi_{0} \otimes \sum \phi_{k}^{\prime}+\psi_{1} \otimes \sum \chi_{k}^{\prime}, \\
& \left(\psi_{0}-\psi_{1}\right) \otimes \sum \phi_{k} \stackrel{U}{\longrightarrow} \psi_{0} \otimes \sum \phi_{k}^{\prime \prime}+\psi_{1} \otimes \sum \chi_{k}^{\prime \prime} .
\end{aligned}
$$

In order to exploit the conservation law we take sums and differences of (12) and (13), and obtain

$$
\begin{aligned}
2 \psi_{0} \otimes \sum \phi_{k} \longrightarrow \psi_{0} \otimes & \sum\left(\phi_{k}^{\prime}+\phi_{k}^{\prime \prime}\right) \\
& +\psi_{1} \otimes \sum\left(\chi_{k}^{\prime}+\chi_{k}^{\prime \prime}\right), \\
2 \psi_{1} \otimes \sum \phi_{k} \longrightarrow \psi_{0} \otimes & \sum\left(\phi_{k}^{\prime}-\phi_{k}^{\prime \prime}\right) \\
& +\psi_{1} \otimes \sum\left(\chi_{k}^{\prime}-\chi_{k}^{\prime \prime}\right) .
\end{aligned}
$$

The conservation law now entails that for any $k$ :

$2 \psi_{0} \otimes \phi_{k} \longrightarrow \psi_{0} \otimes\left(\phi_{k}^{\prime}+\phi_{k}^{\prime \prime}\right)+\psi_{1} \otimes\left(\chi_{k-1}^{\prime}+\chi_{k-1}^{\prime \prime}\right)$,

$2 \psi_{1} \otimes \phi_{k} \longrightarrow \psi_{0} \otimes\left(\phi_{k+1}^{\prime}-\phi_{k+1}^{\prime \prime}\right)+\psi_{1} \otimes\left(\chi_{k}^{\prime}-\chi_{k}^{\prime \prime}\right)$.

At this point we wish to make contact with Wigner's work, and so specify that the apparatus carries no units of the conserved quantity. This is implemented by setting $k=0$, and so $\phi=\phi_{0}$. With this stipulation and allowing for the fact that, in general, the final apparatus states may have negative angular momentum values, we combine (16) and (17) to obtain:

$$
\begin{aligned}
&\left(\psi_{0}\right.\left.+\psi_{1}\right) \otimes \phi_{0} \longrightarrow \frac{1}{2} \psi_{0} \otimes\left(\phi_{0}^{\prime}+\phi_{1}^{\prime}+\phi_{0}^{\prime \prime}-\phi_{1}^{\prime \prime}\right) \\
&+\frac{1}{2} \psi_{1} \otimes\left(\chi_{-1}^{\prime}+\chi_{-1}^{\prime \prime}+\chi_{0}^{\prime}-\chi_{0}^{\prime \prime}\right) \\
&\left(\psi_{0}-\psi_{1}\right) \otimes \phi_{0} \longrightarrow \frac{1}{2} \psi_{0} \otimes\left(\phi_{0}^{\prime}-\phi_{1}^{\prime}+\phi_{0}^{\prime \prime}+\phi_{1}^{\prime \prime}\right) \\
& \quad+\frac{1}{2} \psi_{1} \otimes\left(\chi_{-1}^{\prime}+\chi_{-1}^{\prime \prime}-\chi_{0}^{\prime}+\chi_{0}^{\prime \prime}\right)
\end{aligned}
$$


From here it follows, by comparison with (12) and (13), that $\phi_{0}^{\prime \prime}=\phi_{0}^{\prime}, \phi_{1}^{\prime \prime}=-\phi_{1}^{\prime}, \chi_{-1}^{\prime \prime}=\chi_{-1}^{\prime}, \chi_{0}^{\prime \prime}=-\chi_{0}^{\prime}$. Thus

$$
\begin{aligned}
& \left(\psi_{0}+\psi_{1}\right) \otimes \phi_{0} \longrightarrow \\
& \psi_{0} \otimes\left(\phi_{0}^{\prime}+\phi_{1}^{\prime}\right)+\psi_{1} \otimes\left(\chi_{0}^{\prime}+\chi_{-1}^{\prime}\right), \\
& \left(\psi_{0}-\psi_{1}\right) \otimes \phi_{0} \longrightarrow \\
& \quad \psi_{0} \otimes\left(\phi_{0}^{\prime}-\phi_{1}^{\prime}\right)+\psi_{1} \otimes\left(-\chi_{0}^{\prime}+\chi_{-1}^{\prime}\right) .
\end{aligned}
$$

Taking the partial trace over the system's Hilbert space in (201) and (21) yields (mixed) reduced probe states $\rho^{+}$ and $\rho^{-}$respectively. With $\left\{e_{i}\right\}$ an arbitrary orthonormal basis in $\mathcal{K}$,

$$
\rho^{ \pm}:=\operatorname{tr}_{\mathcal{H}}\left(P\left[U\left(\varphi^{ \pm} \otimes \phi_{0}\right)\right]\right)=\sum\left\langle e_{i}\right| P\left[U\left(\varphi^{ \pm} \otimes \phi\right) e_{i}\right\rangle,
$$

where $P\left[U\left(\varphi^{ \pm} \otimes \phi_{0}\right)\right]$ are the orthogonal projections onto the final states, and $\varphi^{ \pm}=\frac{1}{\sqrt{2}}\left(\psi_{0} \pm \psi_{1}\right)$. For $U$ to yield a measurement in the sense of (10), it is required that the reduced states corresponding to two orthogonal initial states are unambiguously distinguishable; that is that they are supported on orthogonal subspaces of $\mathcal{K}$. This is equivalent to the statement that $\operatorname{tr}\left(\rho^{+} \rho^{-}\right)$must vanish, and it readily emerges that

$$
\begin{aligned}
0=\operatorname{tr}\left(\rho^{+} \rho^{-}\right) & =\left(\left\|\phi_{0}^{\prime}\right\|^{2}-\left\|\phi_{1}^{\prime}\right\|^{2}\right)^{2} \\
+ & \left(\left\|\chi_{-1}^{\prime}\right\|^{2}-\left\|\chi_{0}^{\prime}\right\|^{2}\right)^{2}+2\left|\left\langle\phi_{0}^{\prime} \mid \chi_{0}^{\prime}\right\rangle\right|^{2} .
\end{aligned}
$$

Since each term in this sum is non-negative, it follows that they must each vanish individually, and so $\left\|\phi_{0}^{\prime}\right\|^{2}=\left\|\phi_{1}^{\prime}\right\|^{2}$, $\left\|\chi_{-1}^{\prime}\right\|^{2}=\left\|\chi_{0}^{\prime}\right\|^{2}$ and $\left\langle\phi_{0}^{\prime} \mid \chi_{0}^{\prime}\right\rangle=0$. Hence (23) is only satisfied if either $\phi_{0}^{\prime}=\phi_{1}^{\prime}=0$ or $\chi_{-1}^{\prime}=\chi_{0}^{\prime}=0$, since $\phi_{0}^{\prime}$ and $\chi_{0}^{\prime}$ are collinear. There are two scenarios to consider: first, where $\chi_{-1}^{\prime}=\chi_{0}^{\prime}=0$ and the measurement takes the form

$$
\begin{aligned}
& \left(\psi_{0}+\psi_{1}\right) \otimes \phi_{0} \longrightarrow \psi_{0}^{\prime} \otimes\left(\phi_{0}^{\prime}+\phi_{1}^{\prime}\right), \\
& \left(\psi_{0}-\psi_{1}\right) \otimes \phi_{0} \longrightarrow \psi_{0}^{\prime} \otimes\left(\phi_{0}^{\prime}-\phi_{1}^{\prime}\right) .
\end{aligned}
$$

This is the form that Wigner arrives at on his final page (see our Appendix). The second scenario is given by $\phi_{0}^{\prime}=$ $\phi_{1}^{\prime}=0$ where

$$
\begin{gathered}
\left(\psi_{0}+\psi_{1}\right) \otimes \phi_{0} \longrightarrow \psi_{1}^{\prime} \otimes\left(\chi_{0}^{\prime}+\chi_{-1}^{\prime}\right), \\
\left(\psi_{0}-\psi_{1}\right) \otimes \phi_{0} \longrightarrow \psi_{1}^{\prime} \otimes\left(-\chi_{0}^{\prime}+\chi_{-1}^{\prime}\right) .
\end{gathered}
$$

It is now easy to verify the unitarity of the interaction. The measurement property guarantees that $\phi_{0}^{\prime}$ and $\phi_{1}^{\prime}$ have equal (squared) norm, as do $\chi_{0}^{\prime}$ and $\chi_{-1}^{\prime}$, leaving the right hand sides of (24) and (25) orthogonal, and so too (26) and (27). For both scenarios, the final system state is independent of the initial one, and repeatability is clearly violated.

It seems that dropping the requirement of repeatability has allowed for the possibility of an accurate measurement, whereas before this was ruled out by the noncommutativity of $S_{x}$ with $J_{z}$. Furthermore, here Wigner has chosen $\phi$ to be an eigenstate of the conserved quantity with eigenvalue zero, whereas we saw in the previous subsection that he chose $\phi$ to have very many components in order to overcome the limitation imposed by the conservation law. Hence giving up repeatability also seems to take away the size constraint for the apparatus.

However, Wigner points out (and this has also been noted in [5]) that the issue of a measurement limitation due to the conservation law has been transferred from the system to the apparatus, since (as is made evident above) the final apparatus states must be eigenstates of the $x$ component of the apparatus' angular momentum yielding a pointer observable that does not commute with $J_{z}$. It is natural to consider a pointer reading to be an instance of a repeatable measurement, since otherwise there would be no stable record of the measurement (see also 4). Here the WAY-type limitation reappears at the level of the pointer observable, which turns out not to commute with the apparatus' conserved quantity. Hence the Yanase condition appears to be violated necessarily. Wigner, it seems, was moving toward a general no-go result: that if one wishes to have an accurate measurement, both repeatability and the Yanase condition must be abandoned. Indeed this is the case, as shall be proved in the next section.

\section{The WAY Theorem}

\subsection{The work of Araki and Yanase extended}

Araki and Yanase 2 took up the work of Wigner and proved a general theorem which we state and prove in a somewhat extended and sharpened form. We show that for the same conclusion to be drawn the assumption of repeatability can be replaced by the Yanase condition.

Let $L=L_{1} \otimes \mathbf{1}+\mathbf{1} \otimes L_{2}$ denote the conserved quantity and $M$ the operator we wish to measure.

Theorem Let $\mathcal{M}:=\langle\mathcal{K}, U, \phi, Z, f\rangle$ be a measurement of a discrete-spectrum self-adjoint operator $M$ on $\mathcal{H}$, and let $L_{1}$ and $L_{2}$ be bounded self-adjoint operators on $\mathcal{H}$ and $\mathcal{K}$, respectively, such that $\left[U, L_{1} \otimes \mathbf{1}+\mathbf{1} \otimes L_{2}\right]=0$. Assume that $\mathcal{M}$ is repeatable or satisfies the Yanase condition. Then $\left[L_{1}, M\right]=0$.

Proof. We choose orthonormal bases $\left\{\varphi_{\mu \rho}\right\}$ and $\left\{\phi_{\mu \sigma}\right\}$ of eigenstates of $M$ and $Z$, respectively (with $\rho, \sigma$ as degeneracy parameters). The most general unitary coupling $U$ that constitutes a measurement of $M$ then takes the form

$$
\varphi_{\mu \rho} \otimes \phi \stackrel{U}{\longrightarrow} \sum_{\sigma} \varphi_{\mu \rho \sigma}^{\prime} \otimes \phi_{\mu \sigma}
$$

where $\left\{\varphi_{\mu \rho \sigma}^{\prime}\right\}$ in $\mathcal{H}$ is an arbitrary set of states such that $\sum_{\sigma}\left\|\varphi_{\mu \rho \sigma}^{\prime}\right\|^{2}=1$. Implementing the conservation law (given by $[U, L]=0$ ) we may now write the matrix elements of $L$ in the following way:

$$
\left\langle\varphi_{\mu^{\prime} \rho^{\prime}} \otimes \phi \mid L \varphi_{\mu \rho} \otimes \phi\right\rangle=\sum_{\sigma, \sigma^{\prime}}\left\langle\varphi_{\mu^{\prime} \rho^{\prime} \sigma^{\prime}}^{\prime} \otimes \phi_{\mu^{\prime} \sigma^{\prime}} \mid L \varphi_{\mu \rho \sigma}^{\prime} \otimes \phi_{\mu \sigma}\right\rangle .
$$


The additivity of $L$ and the assumption that $\phi$ is normalized entails that (29) can be written

$$
\begin{array}{r}
\left\langle\varphi_{\mu^{\prime} \rho^{\prime}} \mid L_{1} \varphi_{\mu \rho}\right\rangle+\left\langle\varphi_{\mu^{\prime} \rho^{\prime}} \mid \varphi_{\mu \rho}\right\rangle\left\langle\phi \mid L_{2} \phi\right\rangle \\
=\sum_{\sigma, \sigma^{\prime}}\left[\left\langle\varphi_{\mu^{\prime} \rho^{\prime} \sigma^{\prime}}^{\prime} \mid L_{1} \varphi_{\mu \rho \sigma}^{\prime}\right\rangle\left\langle\phi_{\mu^{\prime} \sigma^{\prime}}^{\prime} \mid \phi_{\mu \sigma}^{\prime}\right\rangle+\right. \\
\left.\left\langle\varphi_{\mu^{\prime} \rho^{\prime} \sigma^{\prime}}^{\prime} \mid \varphi_{\mu \rho \sigma}^{\prime}\right\rangle\left\langle\phi_{\mu^{\prime} \sigma^{\prime}} \mid L_{2} \phi_{\mu \sigma}\right\rangle\right] .
\end{array}
$$

By the orthogonality of pointer eigenstates, $\left\langle\phi_{\mu^{\prime} \sigma^{\prime}}^{\prime} \mid \phi_{\mu \sigma}^{\prime}\right\rangle=$ 0 for $\mu \neq \mu^{\prime}$; examination of each of the remaining terms in the sum in the above expression tells us that these vanish if one of the following conditions holds:

(a) $\left\langle\varphi_{\mu^{\prime} \rho^{\prime} \sigma^{\prime}}^{\prime} \mid \varphi_{\mu \rho \sigma}^{\prime}\right\rangle=0$ for $\mu \neq \mu^{\prime}$;

(b) $\left\langle\phi_{\mu^{\prime} \sigma^{\prime}} \mid L_{2} \phi_{\mu \sigma}\right\rangle=0$ for $\mu \neq \mu^{\prime}$.

Condition (a) is satisfied whenever the measurement is repeatable. Condition (b) is satisfied exactly when the eigenspaces of the pointer observable are invariant under the action of $L_{2}$, i.e when $\left[L_{2}, Z\right]=0$, so that the measurement satisfies the Yanase condition. If either of these are satisfied, then the right hand side of (30) is zero, and thus the left hand side must vanish also. Clearly the second term on the left hand side vanishes due to the orthogonality of the eigenstates of $M$, and the first vanishes if and only if $L_{1}$ leaves $M$-eigenspaces invariant, i.e. if and only if $\left[L_{1}, M\right]=0$.

We interpret the theorem as follows: if $\mathcal{M}$ is a measurement of $M$ and $\left[L_{1}, M\right] \neq 0$, then the conservation of $L$ entails that $\mathcal{M}$ must violate both repeatability and the Yanase condition, in accordance with the expectation that emerged in the previous section.

As the proof shows, the commutativity of $M$ with $L_{1}$ follows from the condition (a), which is in fact a weakening of the repeatability requirement as it merely requires the distinguishability of the post-measurement states of the system. Repeatability is obtained by assuming that the $\varphi_{\mu \rho \sigma}^{\prime}$ are eigenvectors of $M$. In [9] it has been shown that the distinguishability of the post measurement object states on one hand and of the post measurement apparatus states on the other are subject to a WAY-type tradeoff relation. There the distinguishability is quantified by a measure of fidelity, and the measurement inaccuracy is manifested by final pointer states having non-maximal fidelity.

We note that a result of the form of the above theorem (i.e. using the weakened form of repeatability or the Yanase condition to derive the commutativity of the observable to be measured with the conserved quantity) has been proved by Beltrametti et al in 1990 [10] for the special case of minimal unitary measurements, for which the spectra of both the measured observable and pointer are nondegenerate.

As noted above, the violation of the Yanase condition can be understood as disallowing accurate and repeatable measurements of the apparatus observable (since this observable is now subject to the same limitations as prescribed by the WAY theorem). We also observed that the repeatability of pointer measurements is required for ensuring stable and reproducible measurement records. Hence, even if repeatability is sacrificed at the object level, it would seem indispensable at the level of the pointer measurement, thereby enforcing fulfillment of the Yanase condition. Thus we argue that no "measurement" violating the Yanase condition may be called a measurement at all. One may talk only of information transfer between system and apparatus and must also consider how this information can be finally extracted. This conclusion applies to the class of pointer observables that are subject to the WAY theorem.

\subsection{Technical developments}

As demonstrated in a footnote in [2], the case of $L_{2}$ being unbounded can be incorporated into the proof in a natural way. This is achieved by using the unitary operators $V(t)=\exp (i t L)$ and $V_{i}(t)=\exp \left(i t L_{i}\right)($ with $i=1,2$, $t \in \mathbb{R}$ ) and noting that $V(t)=V_{1}(t) \otimes V_{2}(t)$. Then one can follow the previous line of proof, replacing the original operators with their exponentiated forms, and exploiting the boundedness of $L_{1}$.

Ghirardi et al 11 have extended the WAY theorem to the case where $L_{1}$ may be unbounded, but all eigenvectors of $M$ are contained in the domain of $L_{1}$. The measurement is still stipulated to be repeatable. They note that their theorem constrains the feasibility of repeatable measurements of a component of the orbital angular momentum observable in the presence of the conservation of another angular momentum component for the system plus apparatus. Yet their extension still does not cover some physically important cases, namely, those involving observables with continuous spectra.

\section{WAY-type Limitation for Approximate Measurements}

Wigner's paper [1] not only demonstrated the strict impossibility of accurate and repeatable measurements given the conservation law, but also delineated a means by which approximate measurements with approximate repeatability could be recovered. It is also the case, as demonstrated by Araki and Yanase, that this positive part of Wigner's example can be extended to a much more general class of observables and conserved quantities. Here we describe further developments in this area, examine WAY-type limitations for approximate measurements, and discuss how approximate repeatability also follows a trade-off relation with the size of the apparatus in certain circumstances. This helps to elucidate further the crucial role of the Yanase condition in discussions of WAY-type limitations to quantum measurements.

In the case where $\left[L_{1}, M\right] \neq 0$, the limitation given by the WAY theorem can thus be re-expressed more quantitatively: There are approximate measurements of $M$, with some degree of approximate repeatability, which satisfy the Yanase condition, but where good approximations are achieved at the price of requiring a large apparatus, quantified by the magnitude of $\left\langle\phi \mid\left(L_{2}\right)^{2} \phi\right\rangle$. 


\subsection{Overview of results}

Yanase 5] derives an "optimal" lower bound for the probability of the measurement failing to be accurate and repeatable; he considers measurements of a spin component $S_{x}$ where the conserved quantity is $S_{z}+J_{z}$, with $J_{z}$ the $z$-component of the apparatus' (unbounded) angular momentum . The pointer observable is chosen so that it commutes with $J_{z}$. In this case, the lower bound for the probability of the apparatus malfunctioning is given by $\left[8\left\langle\phi \mid\left(J_{z}\right)^{2} \phi\right\rangle\right]^{-1}$. This bound was also illustrated by Ghirardi 12 for rotationally invariant Hamiltonians. Yanase's result, though claimed to be "optimal", still only considered terms up to second moments in $\left(J_{z}\right)$, and thus optimality was not proven rigorously. This was pointed out by Ozawa 4] who obtained a sharper, tight bound without approximations.

Ghirardi et al [1] have considered the case where measurement errors arise from the non-orthogonality of the final apparatus states. They consider both "distorting" and "non-distorting" (yet still repeatable) measurements. They derive lower bounds on the probability of the "malfunctioning" of the apparatus, and even consider the role that large apparatus size has in reducing these probabilities. However, they do not establish the necessity of a large apparatus for good measurements; they merely assume that the error probabilities can be made small by increasing the expectation of the square of the apparatus part of the conserved quantity.

\subsection{Ozawa's trade-off inequality}

Ozawa 4] develops an alternative formulation of the WAY theorem. He introduces a measure of noise to quantify measurement inaccuracy, and shows that this has a lower bound that can be decreased provided the variance of the apparatus' conserved quantity is increased. This tradeoff inequality follows as an application of the CauchySchwarz inequality.

Given a measurement $\mathcal{M}$ that is to serve as an approximate determination of an observable $M$, the noise operator is defined as the difference $N:=Z(\tau)-M$, where $Z(\tau)$ represents the Heisenberg-evolved pointer observable after the interaction period $\tau$. A measure of noise is then given as $\epsilon(\varphi)^{2}:=\left\langle\varphi \otimes \phi \mid N^{2} \varphi \otimes \phi\right\rangle \equiv\left\langle N^{2}\right\rangle$. Clearly $\epsilon(\varphi)^{2} \geq(\Delta N)^{2}$. A global measure of error can be provided by taking the supremum over all (normalized) input states $\varphi$ of the quantity $\epsilon(\varphi)^{2}$, i.e. $\epsilon^{2}:=\sup _{\varphi} \epsilon(\varphi)^{2}$. This quantity should be finite for any measurement that would qualify as an approximate determination of $M$. Then the uncertainty relation entails

$$
\epsilon^{2} \geq \epsilon(\varphi)^{2} \geq \frac{1}{4} \frac{\left|\left\langle\left[Z(\tau)-M, L_{1}+L_{2}\right]\right\rangle\right|^{2}}{(\Delta L)^{2}}
$$

where it is found that $(\Delta L)^{2}=\left(\Delta_{\psi} L_{1}\right)^{2}+\left(\Delta_{\phi} L_{2}\right)^{2}$. The measurement is accurate if and only if $\epsilon=0$.

Thus, if the Yanase condition $\left(\left[Z, L_{2}\right]=0\right)$ is satisfied and the interaction obeys the conservation law, then all that remains in the numerator is $\left|\left\langle\left[M, L_{1}\right]\right\rangle\right|^{2}$. If this is zero then there is no lower bound on the measurement accuracy, in accordance with the findings of WAY.

In the case that $\left|\left\langle\left[M, L_{1}\right]\right\rangle\right|^{2}$ is non-zero but finite, then clearly if $(\Delta L)^{2}$ becomes large the lower bound on the inaccuracy decreases. Furthermore, since the initial system state is arbitrary, only by fixing $\phi$ such that $\left(\Delta_{\phi} L_{2}\right)^{2}$ is large may one increase the accuracy of the measurement, thus establishing the necessity of a large apparatus variance for good measurements.

It is also worthwhile investigating the case of a measurement scheme $\mathcal{M}$ that satisfies neither the Yanase condition nor the commutativity condition $\left[M, L_{1}\right]=0$ but is such that the bound on the right hand side of (31) vanishes; thus, $\left[Z(\tau), L_{1}+L_{2}\right]=\left[M, L_{1}\right]=U^{*}\left[Z, L_{2}\right] U$, by the conservation law. This is clearly satisfied if $\mathcal{M}$ happens to be accurate, $\epsilon=0$. Such a measurement scheme allows for perfectly accurate transfer of information from system to apparatus, and demonstrates the necessary failure of the Yanase condition for this to be achieved.

\subsection{Trade-off relation for repeatability}

Ozawa 13 has proved that observables with a continuous spectrum do not admit any repeatable measurements. This holds regardless of whether there are additive conserved quantities or not. In order to describe repeatability properties of measurements of such observables, it is therefore necessary to have notions of approximate repeatability, and methods for quantifying how repeatable a measurement is. One approach to weaken condition (2) [14, 15. We will explain and use this in Sec. 7.2 in the context of a measurement model.

Here we introduce a different intuitive quantification of repeatability that is somewhat similar to the construction of the noise operator. With this we can generically describe how repeatable a measurement is by utilizing a commutation relation with the conserved quantity. We define:

$$
\mu(\varphi)^{2}:=\left\langle\varphi \otimes \phi \mid(M(\tau)-Z(\tau))^{2} \varphi \otimes \phi\right\rangle ;
$$

intuitively if this expectation is small, then the difference between the measured observable and the time-evolved system observable is small, and hence the measurement should display some level of repeatability. A state independent measure of repeatability may thus be defined as $\mu^{2}:=\sup \mu(\varphi)^{2}$, yielding

$$
\mu^{2} \geq \sup _{\varphi} \frac{1}{4} \frac{\left|\left\langle\left[M(\tau)-Z(\tau), L_{1}+L_{2}\right]\right\rangle\right|^{2}}{\left(\Delta_{\varphi} L_{1}\right)^{2}+\left(\Delta_{\phi} L_{2}\right)^{2}} .
$$

If the Yanase condition is satisfied, then $\left[Z(\tau), L_{1}+L_{2}\right]=$ 0 and so

$$
\mu^{2} \geq \sup _{\varphi} \frac{1}{4} \frac{\left|\left\langle\left[M(\tau), L_{1}+L_{2}\right]\right\rangle\right|^{2}}{\left(\Delta_{\varphi} L_{1}\right)^{2}+\left(\Delta_{\phi} L_{2}\right)^{2}}
$$

which demonstrates that good repeatability may also be achieved when $\left(\Delta_{\phi} L_{2}\right)^{2}$ is large. This condition becomes a necessity when $\left[M, L_{1}\right]$ is non-zero. 


\section{6 "WAYs Out"}

If an observable we wish to measure does not commute with an additive conserved quantity, we have seen that one may still obtain perfectly accurate information transfer between system and apparatus despite the WAY theorem. Here we note some realizations in which this is achieved, and show explicitly that these models violate both repeatability and the Yanase condition.

\subsection{Ohira and Pearle}

Ohira and Pearle 6] provide a "WAY-out" of the limitation arising from the WAY theorem via a model in which both the object and the probe are given as spin- $\frac{1}{2}$ systems. The measurement coupling is generated by a rotationally invariant Hamiltonian of the form $H=(\mathbf{S}+\mathbf{J}) \cdot(\mathbf{S}+\mathbf{J})$.

We proceed under the notation that $\psi_{ \pm}$represent both $S_{z}$ and $J_{z}$ eigenstates, and $\phi=\psi_{+}$. The evolution takes the form (with the interaction period $\tau=\pi / 2$ ):

$$
\begin{array}{ll}
\left(\psi_{+}+\psi_{-}\right) \otimes \phi \longrightarrow & \left(-\psi_{+}\right) \otimes\left(\psi_{+}+\psi_{-}\right), \\
\left(\psi_{+}-\psi_{-}\right) \otimes \phi \longrightarrow & \left(-\psi_{+}\right) \otimes\left(\psi_{+}-\psi_{-}\right) .
\end{array}
$$

Here the appropriate pointer observable is $Z=J_{x}$. This model is not repeatable, and also violates the Yanase condition.

Recalling equations (24) and (25) which appeared on Wigner's final page, we see that these have precisely the same form as (35), apart from an inconsequential difference of initial pointer states.

Our analysis of this model of Ohira and Pearle coincides with that of Wigner's last page (Sec. 3.2). They point out that this model has demonstrated that if repeatability is not insisted upon, one may achieve an accurate measurement despite the restrictions of the WAY theorem. However, as we have seen, the theorem does not stipulate any limitation to the accuracy (of information transfer) when both the repeatability and Yanase conditions are violated, as is the case here. This is precisely the setting in which perfect accuracy is achievable, and this model of Ohira and Pearle is therefore fully in accordance with the WAY theorem as we have given it.

Ohira and Pearle's aim was to expose and correct a common misreading of the WAY theorem as prohibiting accurate measurements in the presence of an additive conserved quantity. This prohibition, they show, is removed at the expense of giving up the repeatability of the measurement. We know now that in addition the Yanase condition has to be violated as well.

Ozawa's inequality (31) shows how the zero-error measurement can be achieved; the condition for vanishing lower bound for the error takes the form $U^{*}\left[Z, L_{2}\right] U=\left[M, L_{1}\right]$. In this model, it is easily verified that $U^{*} \mathbf{1} \otimes S_{y} U=S_{y} \otimes \mathbf{1}$, which indeed entails that the expectation value in the numerator of Ozawa's inequality vanishes.

\subsection{The SWAP Map Example}

Following the work of Wigner and Ohira and Pearle, we note that these "WAYs out" are both examples of a remarkably simple structure. They violate both repeatability and the Yanase condition, and whenever the initial system state is an eigenstate of the observable to be measured, both take the form of an unentangled (product) state after the unitary interaction. It is known [16] that the only non-entangling unitary operators $U$ on $\mathcal{H}_{1} \otimes \mathcal{H}_{2}$ are either of the form: (i) $U(\varphi \otimes \phi)=(V \varphi) \otimes(W \phi)$ (with $V$ and $W$ unitary on $\mathcal{H}_{1}$ and $\mathcal{H}_{2}$ respectively), or (ii) $U(\varphi \otimes \phi)=\left(V_{21} \phi\right) \otimes\left(W_{12} \varphi\right)$ with $V_{21}: \mathcal{H}_{2} \rightarrow \mathcal{H}_{1}$ and $W_{12}: \mathcal{H}_{1} \rightarrow \mathcal{H}_{2}$ surjective isometries. This latter scenario is only possible if $\operatorname{dim} \mathcal{H}_{1}=\operatorname{dim} \mathcal{H}_{2}$ (with the dimension possibly infinite).

One of the simplest examples of a non-entangling unitary map (which is of type (ii), see above) is provided by the $S W A P$ map $U_{S}$ on $\mathcal{H} \otimes \mathcal{H}$, defined by $U_{S}(\varphi \otimes \phi)=\phi \otimes \varphi$. If this unitary map is to be used in the context of a measurement, we see that (1) takes the form $\langle\varphi \mid \mathrm{E}(X) \varphi\rangle=$ $\left\langle\varphi \mid \mathrm{E}^{Z}\left(f^{-1}(X)\right) \varphi\right\rangle$ (for all $\varphi \in \mathcal{H}$ ), which can be satisfied if $\mathrm{E}=\mathrm{E}^{M}=\mathrm{E}^{Z}$, and hence $Z=M$. This also respects any conservation law that is additive and where each non-trivial operator in the sum takes the same form. The noise operator is given as $N=U^{*}(\mathbf{1} \otimes Z) U-M \otimes \mathbf{1}=$ $Z \otimes \mathbf{1}-M \otimes \mathbf{1}$. Thus, since we have chosen $Z=M$, the noise operator $N$ vanishes and we have a perfectly accurate information transfer between system and apparatus. However, as the SWAP map violates the Yanase condition, there remains the problem of recovering this information from the pointer observable.

\section{Position Measurements Obeying Momentum Conservation}

Many of the observables that make up a coherent and complete view of (quantum) physical reality are not of the class that have been discussed thus far. Technical difficulties arise in the context of unbounded operators with continuous spectrum, position and (linear) momentum being two noteworthy examples. However if one wishes for a comprehensive understanding of WAY-type limitations to the measurability of physical quantities, it is critical to understand the fundamental case of position measurements that obey momentum conservation. In this section we discuss some results that have been obtained in this context. Any WAY-type theorem for these observables will have to take into account Ozawa's result that as a continuous quantity, position cannot be measured repeatably.

In 17] the present authors have provided strong evidence for the existence for such a theorem in the positionmomentum case. They demonstrate that a model put forward by Ozawa claiming to demonstrate no WAY-type restriction is flawed. The model of Ozawa satisfies the Yanase condition, and one can show that only in the limit of the pointer preparation becoming a delta-function may the inaccuracy tend to zero, which comes at the expense 
of the apparatus' momentum distribution having a large width (suitably defined). Furthermore [17] provides a model that explicitly violates the Yanase condition, where arbitrarily accurate and repeatable measurements may still be achieved without resorting to a size constraint on the apparatus.

\subsection{A General Argument}

It is again possible to implement the Ozawa inequality (31) to obtain a general argument in favour of WAY-limitations in the continuous unbounded case when the Yanase condition is satisfied. The form of the position-momentum commutator allows the supremum on the right-hand side of (31) to be taken in the following way:

$$
\epsilon^{2} \geq \frac{1}{4} \frac{1}{\inf _{\varphi}\left(\Delta_{\varphi} P\right)^{2}+\left(\Delta_{\phi} P_{\mathcal{A}}\right)^{2}}=\frac{1}{4\left(\Delta_{\phi} P_{\mathcal{A}}\right)^{2}} .
$$

with $\left(\Delta_{\varphi} P\right)^{2}$ and $\left(\Delta_{\phi} P_{\mathcal{A}}\right)^{2}$ the variance of the momentum in the system and apparatus respectively. This bound allows for an increase in accuracy only when $\left(\Delta_{\phi} P_{\mathcal{A}}\right)^{2}$ is large, establishing the necessity of large apparatus size for good measurements.

Precisely the same bound arises when one considers the repeatability (defined in (33));

$$
\mu^{2} \geq \frac{1}{4\left(\Delta_{\phi} P_{\mathcal{A}}\right)^{2}} .
$$

This provides an indication that good repeatability can indeed be achieved if (and only if) there is a large momentum variance in the probe.

Notice that the non-zero lower bounds to both accuracy and repeatability arise after explicit implementation of the Yanase condition, $\left[Z, P_{\mathcal{A}}\right]=0$. If we relinquish this condition, there is nothing that would prevent $\left[Z(\tau)-Q, P+P_{\mathcal{A}}\right]$ from vanishing. Indeed this would be the case in any model where one could choose the pointer observable as the apparatus' position, $Q_{\mathcal{A}}$.

In the position-momentum case, the role of the Yanase condition must be considered very carefully. Previously (in the case where the WAY theorem certainly applied) we argued for the Yanase condition by applying the WAY theorem to the measurement of the pointer, of which we demanded accurate and repeatable measurements. However, since no such theorem has been proven in the continuous/unbounded case, one must be more tentative when stipulating this condition, and it may be considered as a precautionary manoeuvre. The models discussed in [17, as well as the above model-independent relations point in the direction of a WAY-type limitation if the Yanase condition is satisfied and no such obstruction if it is not.

The last conclusion (of "no obstruction") contrasts, perhaps somewhat surprisingly, the WAY theorem for accurate measurements: Within the realm of that theorem, it is not sufficient to violate the Yanase condition in order to lift the obstruction against perfect accuracy and repeatability. The fact that no size constraint is required for good measurements of position if the pointer observable is a position itself can be understood by considering the lower bounds in equations (31) and (33): If the object position does not change during the interaction, $M(t)=M=Q$, and the pointer is $Z=Q_{\mathcal{A}}$, the lower bounds become zero in both cases since the commutator of the noise operator $N=Q_{\mathcal{A}}(t)-Q$ with the conserved quantity $L_{1}+L_{2}=$ $P+P_{\mathcal{A}}$ vanishes identically. This is a consequence of the fact that $\left[Q_{\mathcal{A}}(t), P+P_{\mathcal{A}}\right]=i \mathbf{1}=[Q, P]$. Such cancellation of commutators living on different Hilbert spaces can only arise for pairs of observables with constant commutators.

It is not known whether, under violation of the Yanase condition, there exist measurements of position that are fully accurate, and repeatable to a good approximation. It is also an open problem whether, again with giving up the Yanase condition, approximate spin measurements obeying angular momentum conservation are possible with good repeatability properties, without any constraint on the size of the apparatus.

\subsection{The Problem of Stein and Shimony}

In 1979 Stein and Shimony [7] posed a problem concerning the possibility of realizing a two-valued (and hence coarse-grained) position measurement that respects the conservation of momentum.

This problem takes the form of whether there exists a non-zero function $\phi \in L^{2}(\mathbb{R})$ and unitary operator $U$ : $L^{2}\left(\mathbb{R}^{2}\right) \rightarrow L^{2}\left(\mathbb{R}^{2}\right)$ that commutes with the shift operators (defined by $T_{t}(g)(x, y)=g(x+t, y+t)$ for $g \in L^{2}\left(\mathbb{R}^{2}\right)$ and $t \in \mathbb{R})$ ) and satisfy:

$$
\begin{aligned}
& \operatorname{supp}[U(\varphi \otimes \phi)] \subseteq \mathbb{R}^{+} \times \mathbb{R}^{+} \text {if } \operatorname{supp} \varphi \subseteq \mathbb{R}^{+}, \\
& \operatorname{supp}[U(\varphi \otimes \phi)] \subseteq \mathbb{R}^{-} \times \mathbb{R}^{-} \text {if } \operatorname{supp} \varphi \subseteq \mathbb{R}^{-},
\end{aligned}
$$

where $\varphi \in L^{2}(\mathbb{R})$. With the pointer being a two-valued, discretized position observable, this coupling necessarily violates the Yanase condition. The condition that the unitary $U$ commutes with $T_{t}$ is a mathematical expression of the conservation of the total momentum $P+P_{\mathcal{A}}$.

Here we provide a position measurement scheme [15] that approximately satisfies the above requirements with the quality of the approximation becoming arbitrarily good as the value of the coupling parameter $\lambda$ becomes large. The momentum-conserving unitary operator $U$ which describes the interaction is given by

$$
U=\exp \left[-i \frac{\lambda}{2}\left(\left(Q-Q_{\mathcal{A}}\right) P_{\mathcal{A}}+P_{\mathcal{A}}\left(Q-Q_{\mathcal{A}}\right)\right)\right],
$$

where for example we have written $\left(Q-Q_{\mathcal{A}}\right) P_{\mathcal{A}}$ as shorthand for $\left(Q \otimes \mathbf{1}-\mathbf{1} \otimes Q_{\mathcal{A}}\right) \mathbf{1} \otimes P_{\mathcal{A}}$. The pointer observable is given as $Q_{\mathcal{A}}$, and the measured observable $\mathrm{E}$ [Eq. (1)] is of the form $\mathrm{E}(X)=\chi_{X} \star e(Q)$, if the scaling function $f$ is chosen such that $f^{-1}(X)=\left(1-e^{-\lambda}\right) X$. Here $\chi_{X}$ represents the characteristic set function. The probability density $e=e^{(\lambda)}$ depends on $\lambda$ in the following way:

$$
e^{(\lambda)}(q)=\left(e^{\lambda}-1\right)\left|\phi\left(-q\left(e^{\lambda}-1\right)\right)\right|^{2} .
$$


In order to answer the question of Stein and Shimony, we first recast the conditions that need to be satisfied as follows. Firstly, the measurement must satisfy a stronger form of the probability reproducibility condition called the calibration condition, which requires that if the initial state is localized in the positive (or negative) half line, then this result is shown on the pointer with certainty. We shall denote the spectral measures of $Q$ and $Q_{\mathcal{A}}$ by $\mathrm{Q}$ and $Q_{\mathcal{A}}$ respectively. Allowing for some error, this may be written (for $\alpha>0$ )

$$
\left\langle\Psi_{\tau} \mid \mathbf{1} \otimes Q_{\mathcal{A}}[-\alpha, \infty) \Psi_{\tau}\right\rangle=1
$$

if $\operatorname{supp} \varphi \subseteq[0, \infty)$, and we show that $[-\alpha, \infty)$ can become arbitrarily close to $[0, \infty)$ if $\lambda$ is made suitably large.

The second requirement is that of repeatability, which we give as a slightly modified version of (2) whereby the immediate subsequent measurement is of the observable $Q$. This takes the form (with $\beta>0$ )

$$
\begin{aligned}
\left\langle\Psi_{\tau} \mid \mathrm{Q}[-\beta, \infty) \otimes \mathrm{Q}_{\mathcal{A}}\left(\mathbb{R}^{+}\right) \Psi_{\tau}\right\rangle= & \\
\left\langle\Psi_{\tau} \mid \mathbf{1} \otimes \mathrm{Q}_{\mathcal{A}}\left(\mathbb{R}^{+}\right) \Psi_{\tau}\right\rangle & =\left\langle\varphi \mid \mathrm{E}\left(\mathbb{R}^{+}\right) \varphi\right\rangle,
\end{aligned}
$$

where the last equality results from the probability reproducibility condition. We shall show that this may be satisfied for all $\varphi$ and that $\beta$ can be made arbitrarily small.

We shall make the immediate specification that the initial state wave function $\phi$ of the apparatus be supported on a fixed finite interval of width $2 \ell$ around the origin; $\operatorname{supp} \phi=[-\ell, \ell]$. Therefore the distribution $e^{(\lambda)}$ is supported on the $\lambda$-scaled interval $[-\delta, \delta]$, with $\delta=\ell /\left(e^{\lambda}-1\right)$.

After some manipulation the calibration requirement (40) takes the form

$$
\int_{0}^{\infty}|\varphi(q)|^{2} \chi_{\left[-\alpha^{\prime}, \infty\right)} * e^{(\lambda)}(q) d q=1
$$

with $\alpha^{\prime}=f(\alpha)$. Thus we require $\chi_{\left[-\alpha^{\prime}, \infty\right)} * e^{(\lambda)}(q)=1$ for all $q \geq 0$ and so

$$
\int_{-\infty}^{\alpha^{\prime}+q} e^{(\lambda)}(y) d y=1
$$

which is satisfied if $q \geq \delta-\alpha^{\prime}$. The smallest $\alpha^{\prime}$ consistent with this constraint occurs when $\alpha^{\prime}=\delta$, and so $\alpha=\ell e^{-\lambda}$. Therefore we see that indeed $\alpha \rightarrow 0$ as $\lambda \rightarrow \infty$. It must also be shown that the same behaviour emerges in the case when $\operatorname{supp} \varphi \subseteq(-\infty, 0]$ but we omit this essentially identical calculation, and this completes the proof.

We now address the repeatability requirement. Writing (41) in integral form and rearranging, we see that

$$
\int|\varphi(q)|^{2}\left(\chi_{[-\beta, \infty)}(q)-1\right) \chi_{[0, \infty)} * e^{(\lambda)}(q) d q=0,
$$

and so

$$
\chi_{(-\infty,-\beta)}(q) \int_{-\infty}^{q} e^{(\lambda)}(y) d y=0 .
$$

This expression certainly vanishes if $q \geq-\beta$. When $q<$ $-\beta$, recalling that $\operatorname{supp} e^{(\lambda)}=[-\delta, \delta]$, we see that if $-\delta \geq$ $-\beta$ (and thus $\beta \geq \delta$ ) then the integral vanishes. Since we are looking for the smallest $\beta$ for which this may be satisfied, we choose $\beta=\delta=\ell /\left(e^{\lambda}-1\right)$. Therefore in the large $\lambda$ limit, $\beta$ is arbitrarily small, showing that arbitrarily good repeatability may be achieved. Due to the symmetry of the support of $e^{(\lambda)}$, it follows that arbitrarily good repeatability holds also for the $\mathbb{R}^{-}$outcome on the pointer.

Although this model provides only an approximate solution to the problem of Stein and Shimony, we note that from an operational perspective this does not differ from an exact solution. Since the accuracy and approximate repeatability can be made arbitrarily good by simply tuning the coupling parameter, in any experimental realization this could not be distinguished from a measurement in which perfect accuracy and repeatability can be achieved. This does not require a large momentum spread in the probe, and it has been shown that the present model indeed presents an approximate measurement scheme for the full position observable $Q$, with arbitrarily good accuracy and repeatability properties [17.

\section{Concluding Remarks}

The WAY theorem, with its generalizations, is applicable to a large class of physically important scenarios. In any situation in which, for example, spin or angular momentum is the relevant observable, the measurement accuracy is likely to be hampered by a WAY-type constraint. When considering the manipulation of individual quantum objects using other small objects as 'apparatus', it may not be possible to fulfill the requirement of large variance of the apparatus part of the conserved quantity. Such scenarios do occur in quantum information processing and quantum control. Ozawa and coworkers [18 have in fact demonstrated a limitation to the realizability of quantum logic gates insofar as the observables involved are subject to the WAY theorem. This has led to an increased awareness that attention has to be paid to the presence of conserved quantities in the design of quantum gates.

In the case of position measurements that obey momentum conservation, no WAY-type obstruction exists if one asks only for a measurement of the relative distance between the object and a "reference system". In this case, when the reference system is provided by part of the apparatus, the measured observable can be given as the relative position. As is alluded to in [19, it appears that there is a link to the theory of superselection rules and quantum reference frames (see, e.g. [20]), which has been the subject of much interest and investigation recently. This possible link opens up an avenue that requires further systematic study.

Acknowledgments. Thanks are due to Rebecca Ronke and Tom Potts for many helpful discussions and careful reading of drafts of this manuscript. This work was supported by EPSRC UK. 


\section{Appendix: Reconstructing Wigner's last page}

In this appendix we shall carefully reconstruct the argument that appears on the final page of Wigner's 1952 paper 1]. Although Wigner's work is succinct and simple, the lack of detailed calculations makes reproducing his conclusions somewhat harder work than one might imagine. We also present some subtly different arguments from those found in the original work.

Wigner restricts his consideration to the case where the post-interaction states are of product form (unentangled) in the system-apparatus Hilbert space, and he makes the choice that the initial apparatus state $\phi$ be an eigenstate of $S_{z}$ with eigenvalue zero. He writes

$$
\begin{aligned}
& \left(\psi_{0}+\psi_{1}\right) \otimes \phi \longrightarrow \sum_{i=0}^{1} \psi_{i}^{\prime} \otimes \sum \phi_{j}^{\prime}, \\
& \left(\psi_{0}-\psi_{1}\right) \otimes \phi \longrightarrow \sum_{i=0}^{1} \psi_{i}^{\prime \prime} \otimes \sum \phi_{j}^{\prime \prime},
\end{aligned}
$$

with $\psi_{i}^{\prime}$ and $\psi_{i}^{\prime \prime}$ representing un-normalized $S_{z}$ eigenstates. In order that Wigner's analysis be compelling, we must assume $\phi_{j}^{\prime}$ and $\phi_{j}^{\prime \prime}$ to be eigenstates of the apparatus' angular momentum, $J_{z}$. The reason for this choice will become clear shortly; this is the only way in which consistency with the conservation law can be maintained. We omit summation indices on the apparatus Hilbert space since it is assumed to run to infinity. However, the number of non-zero terms in this expansion is dramatically reduced due to the choice of initial apparatus state and the conservation law; the left hand side of (46) contains a superposition of $S_{z}$ eigenstates, and thus a superposition of states containing zero and one "unit" of the conserved quantity. The right hand side cannot, then, contain more than one such unit.

In order to correspond to Wigner's analysis, we proceed under the restriction that 0 be the lowest eigenvalue for the apparatus' conserved quantity, and from here it follows that (46) and (47) take on a much simpler forms. With $\phi=\phi_{0}$ and dropping all terms with the apparatus containing two or more units of the conserved quantity, we have

$$
\left(\psi_{0}+\psi_{1}\right) \otimes \phi_{0} \longrightarrow \psi_{0}^{\prime} \otimes \phi_{0}^{\prime}+\psi_{0}^{\prime} \otimes \phi_{1}^{\prime}+\psi_{1}^{\prime} \otimes \phi_{0}^{\prime}+\psi_{1}^{\prime} \otimes \phi_{1}^{\prime},
$$

$$
\left(\psi_{0}-\psi_{1}\right) \otimes \phi_{0} \longrightarrow \psi_{0}^{\prime \prime} \otimes \phi_{0}^{\prime \prime}+\psi_{0}^{\prime \prime} \otimes \phi_{1}^{\prime \prime}+\psi_{1}^{\prime \prime} \otimes \phi_{0}^{\prime \prime}+\psi_{1}^{\prime \prime} \otimes \phi_{1}^{\prime \prime},
$$

Indeed, the conservation law provides an even stronger restriction, and the last term on the right hand side of (48) must in fact be zero, and thus at least one of $\psi_{1}^{\prime}$ and $\phi_{1}^{\prime}$ must always vanish. The same argument applies to (49) and so (independently), at least one of $\psi_{1}^{\prime \prime}$ and $\phi_{1}^{\prime \prime}$ must vanish too.

It follows from (48) and consistency with the conservation law that $\psi_{0}^{\prime}$ and $\phi_{0}^{\prime}$ are necessarily non-zero. For if either did vanish, the right hand side would contain one unit of the conserved quantity with certainty, and the left hand side only with probability $\frac{1}{2}$. The same argument runs in clear analogy for the double-primed quantities. There are then four scenarios that require consideration:

Case 1: $\psi_{1}^{\prime} \neq 0, \phi_{1}^{\prime}=0, \psi_{1}^{\prime \prime} \neq 0, \phi_{1}^{\prime \prime}=0$;

Case 2: $\psi_{1}^{\prime} \neq 0, \phi_{1}^{\prime}=0, \phi_{1}^{\prime \prime} \neq 0, \psi_{1}^{\prime \prime}=0$;

Case 3: $\phi_{1}^{\prime} \neq 0, \psi_{1}^{\prime}=0, \psi_{1}^{\prime \prime} \neq 0, \phi_{1}^{\prime \prime}=0$;

Case $4: \phi_{1}^{\prime} \neq 0, \psi_{1}^{\prime}=0, \phi_{1}^{\prime \prime} \neq 0, \psi_{1}^{\prime \prime}=0$.

With this in mind, one can add (48) and (49) to give the evolution of $\psi_{0} \otimes \phi_{0}$ :

$$
\begin{aligned}
& 2 \psi_{0} \otimes \phi_{0} \longrightarrow \psi_{0}^{\prime} \otimes \phi_{0}^{\prime}+\psi_{0}^{\prime} \otimes \phi_{1}^{\prime}+\psi_{1}^{\prime} \otimes \phi_{0}^{\prime}+ \\
& \psi_{0}^{\prime \prime} \otimes \phi_{0}^{\prime \prime}+\psi_{0}^{\prime \prime} \otimes \phi_{1}^{\prime \prime}+\psi_{1}^{\prime \prime} \otimes \phi_{0}^{\prime \prime},
\end{aligned}
$$

and for the evolution of $\psi_{1} \otimes \phi$ we subtract:

$$
\begin{aligned}
2 \psi_{1} \otimes \phi_{0} \longrightarrow & \psi_{0}^{\prime} \otimes \phi_{0}^{\prime}+\psi_{0}^{\prime} \otimes \phi_{1}^{\prime}+\psi_{1}^{\prime} \otimes \phi_{0}^{\prime}- \\
& \psi_{0}^{\prime \prime} \otimes \phi_{0}^{\prime \prime}-\psi_{0}^{\prime \prime} \otimes \phi_{1}^{\prime \prime}-\psi_{1}^{\prime \prime} \otimes \phi_{0}^{\prime \prime} .
\end{aligned}
$$

We first consider Case 1 where (50) and (51) reduce to

$$
2 \psi_{0} \otimes \phi_{0} \longrightarrow \psi_{0}^{\prime} \otimes \phi_{0}^{\prime}+\psi_{1}^{\prime} \otimes \phi_{0}^{\prime}+\psi_{0}^{\prime \prime} \otimes \phi_{0}^{\prime \prime}+\psi_{1}^{\prime \prime} \otimes \phi_{0}^{\prime \prime},
$$

and

$$
2 \psi_{1} \otimes \phi_{0} \longrightarrow \psi_{0}^{\prime} \otimes \phi_{0}^{\prime}+\psi_{1}^{\prime} \otimes \phi_{0}^{\prime}-\psi_{0}^{\prime \prime} \otimes \phi_{0}^{\prime \prime}-\psi_{1}^{\prime \prime} \otimes \phi_{0}^{\prime \prime}
$$

Since the left hand side of (52) contains no units of the conserved quantity, so must the right, and therefore $\psi_{1}^{\prime} \otimes$ $\phi_{0}^{\prime}=-\psi_{1}^{\prime \prime} \otimes \phi_{0}^{\prime \prime}$. Similarly in (53) the left hand side contains one unit, and if the right hand side is to agree, we require that $\psi_{0}^{\prime} \otimes \phi_{0}^{\prime}=\psi_{0}^{\prime \prime} \otimes \phi_{0}^{\prime \prime}$.

With $\psi_{1}^{\prime} \otimes \phi_{0}^{\prime}=-\psi_{1}^{\prime \prime} \otimes \phi_{0}^{\prime \prime}$ we get:

$$
2 \psi_{0} \otimes \phi_{0} \longrightarrow \psi_{0}^{\prime} \otimes \phi_{0}^{\prime}+\psi_{0}^{\prime \prime} \otimes \phi_{0}^{\prime \prime},
$$

and thus, with $\psi_{0}^{\prime} \otimes \phi_{0}^{\prime}=\psi_{0}^{\prime \prime} \otimes \phi_{0}^{\prime \prime}$,

$$
\psi_{0} \otimes \phi_{0} \longrightarrow \psi_{0}^{\prime} \otimes \phi_{0}^{\prime}
$$

Also,

$$
2 \psi_{1} \otimes \phi_{0} \longrightarrow \psi_{1}^{\prime} \otimes \phi_{0}^{\prime}-\psi_{1}^{\prime \prime} \otimes \phi_{0}^{\prime \prime},
$$

and finally, exploiting the condition $\psi_{1}^{\prime} \otimes \phi_{0}^{\prime}=-\psi_{1}^{\prime \prime} \otimes \phi_{0}^{\prime \prime}$, we arrive at

$$
\psi_{1} \otimes \phi_{0} \longrightarrow \psi_{1}^{\prime} \otimes \phi_{0}^{\prime} .
$$

We now consider Case 2 which, with (50) gives

$$
2 \psi_{0} \otimes \phi_{0} \longrightarrow \psi_{0}^{\prime} \otimes \phi_{0}^{\prime}+\psi_{1}^{\prime} \otimes \phi_{0}^{\prime}+\psi_{0}^{\prime \prime} \otimes \phi_{0}^{\prime \prime}+\psi_{0}^{\prime \prime} \otimes \phi_{1}^{\prime \prime}
$$

and thus one might wish to conclude that $\psi_{1}^{\prime} \otimes \phi_{0}^{\prime}=$ $-\psi_{0}^{\prime \prime} \otimes \phi_{1}^{\prime \prime}$. However, this can never be satisfied; these vectors must be distinct unless they are both zero (which is excluded, by assumption), since the unit of conserved quantity resides in different Hilbert spaces. 
Case 3 gives

$$
2 \psi_{0} \otimes \phi_{0} \longrightarrow \psi_{0}^{\prime} \otimes \phi_{0}^{\prime}+\psi_{0}^{\prime} \otimes \phi_{1}^{\prime}+\psi_{0}^{\prime \prime} \otimes \phi_{0}^{\prime \prime}+\psi_{1}^{\prime \prime} \otimes \phi_{0}^{\prime \prime}
$$

and we conclude that it must be the case that $\psi_{0}^{\prime} \otimes \phi_{1}^{\prime}=$ $-\psi_{1}^{\prime \prime} \otimes \phi_{0}^{\prime \prime}$ which, again, cannot be fulfilled for both nonzero. We therefore must also reject Case 3 .

Finally Case 4 gives

$$
2 \psi_{0} \otimes \phi_{0} \longrightarrow \psi_{0}^{\prime} \otimes \phi_{0}^{\prime}+\psi_{0}^{\prime} \otimes \phi_{1}^{\prime}+\psi_{0}^{\prime \prime} \otimes \phi_{0}^{\prime \prime}+\psi_{0}^{\prime \prime} \otimes \phi_{1}^{\prime \prime}
$$

and

$$
2 \psi_{1} \otimes \phi_{0} \longrightarrow \psi_{0}^{\prime} \otimes \phi_{0}^{\prime}+\psi_{0}^{\prime} \otimes \phi_{1}^{\prime}-\psi_{0}^{\prime \prime} \otimes \phi_{0}^{\prime \prime}-\psi_{0}^{\prime \prime} \otimes \phi_{1}^{\prime \prime},
$$

and so $\psi_{0}^{\prime} \otimes \phi_{1}^{\prime}=-\psi_{0}^{\prime \prime} \otimes \phi_{1}^{\prime \prime}$ and $\psi_{0}^{\prime} \otimes \phi_{0}^{\prime}=\psi_{0}^{\prime \prime} \otimes \phi_{0}^{\prime \prime}$.

It is now evident that each of the permissible cases gives the same state evolution for $\psi_{0} \otimes \phi$; Case 4 yields

$$
2 \psi_{0} \otimes \phi_{0} \longrightarrow \psi_{0}^{\prime} \otimes \phi_{0}^{\prime}+\psi_{0}^{\prime \prime} \otimes \phi_{0}^{\prime \prime},
$$

and with $\psi_{0}^{\prime} \otimes \phi_{0}^{\prime}=\psi_{0}^{\prime \prime} \otimes \phi_{0}^{\prime \prime}$, we arrive at

$$
\psi_{0} \otimes \phi_{0} \longrightarrow \psi_{0}^{\prime} \otimes \phi_{0}^{\prime} .
$$

However, for the evolution of $\psi_{1} \otimes \phi$, using $\psi_{0}^{\prime} \otimes \phi_{1}^{\prime}=$ $-\psi_{0}^{\prime \prime} \otimes \phi_{1}^{\prime \prime}$, we see that a different form emerges than from Case 1:

$$
\psi_{1} \otimes \phi_{0} \longrightarrow \psi_{0}^{\prime} \otimes \phi_{1}^{\prime} .
$$

With these considerations, we now summarise the possible forms of the evolution of $\left(\psi_{0}+\psi_{1}\right) \otimes \phi_{0}$ and $\left(\psi_{0}-\right.$ $\left.\psi_{1}\right) \otimes \phi_{0}$. Remembering that the only cases which contain, a priori, no contradiction, are Cases 1 and 4, the first scenario is that Case 1 is satisfied, and we have:

$$
\left(\psi_{0}+\psi_{1}\right) \otimes \phi_{0} \longrightarrow\left(\psi_{0}^{\prime}+\psi_{1}^{\prime}\right) \otimes \phi_{0}^{\prime},
$$

and

$$
\left(\psi_{0}-\psi_{1}\right) \otimes \phi_{0} \longrightarrow\left(\psi_{0}^{\prime}-\psi_{1}^{\prime}\right) \otimes \phi_{0}^{\prime} .
$$

This cannot represent a measurement in any ordinary or physically meaningful sense, since the final states of the apparatus coincide on the right hand side of (65) and (66), leaving us in the position that there is no way of distinguishing which eigenstate of $S_{x}$ had been present on the left hand side. Furthermore, this product form does not correspond to a modification of equations (6) and (7) (as is claimed by Wigner).

The second scenario is that Case 4 is satisfied, and we see that summing (57) with (63) gives:

$$
\left(\psi_{0}+\psi_{1}\right) \otimes \phi_{0} \longrightarrow \psi_{0}^{\prime} \otimes\left(\phi_{0}^{\prime}+\phi_{1}^{\prime}\right)
$$

and subtracting:

$$
\left(\psi_{0}-\psi_{1}\right) \otimes \phi_{0} \longrightarrow \psi_{0}^{\prime} \otimes\left(\phi_{0}^{\prime}-\phi_{1}^{\prime}\right)
$$

This coincides with (24) and (25) (Sec. 3.2), and is the same result as Wigner obtained on his final page.

\section{References}

1. E. Wigner, Z. Phys. 133, 101 (1952).

2. H. Araki, M.M. Yanase, Phys. Rev. 120, 622 (1960).

3. E. Wigner, Measurement of quantum mechanical operators. Translation of 1]; http://arxiv.org/abs/1012.4372.

4. M. Ozawa, Phys. Rev. Lett. 88, 050402 (2002).

5. M.M. Yanase, Phys. Rev. 123, 666 (1961).

6. T. Ohira, P. Pearle, Am. J. Phys. 56, 692 (1988).

7. A. Shimony, H. Stein, The American Mathematical Monthly 86, No. 4 (Apr., 1979), pp. 292-293.

8. P. Busch, P. Lahti, P. Mittelstaedt, The Quantum Theory of Measurement, Springer, Berlin, 1991, 2nd. ed. 1996.

9. T. Miyadera, H. Imai, Phys. Rev. A74, 024101 (2006).

10. E.G. Beltrametti, G. Cassinelli, P.J. Lahti, J. Math. Phys. 31, 91 (1990)

11. G.C. Ghirardi, A. Rimini, T. Weber, J. Math. Phys. 24, 2454 (1983).

12. G.C. Ghirardi, F. Miglietta, A. Rimini, T. Weber, Phys. Rev. D 24347 (1981); 24, 353 (1981).

13. M. Ozawa, J. Math. Phys. 25, 79 (1984).

14. E.B. Davies, J. Func. An. 6, 318-346 (1970).

15. P. Busch, M. Grabowski, P. Lahti, Operational Quantum Physics, Springer, Berlin, 1995/1997.

16. P. Busch, Int. J. Theor. Phys. 42, 5 (2003).

17. P. Busch, L. Loveridge, Phys. Rev. Lett., in press (2011). http://arxiv.org/abs/1005.0569.

18. M. Ozawa, Phys. Rev. Lett. 89, 057902 (2002); T. Karasawa, J. Gea-Banacloche, M. Ozawa, J. Phys. A: Math. Theor. 42, 225303 (2009).

19. Y. Aharonov, D. Rohrlich, Quantum Paradoxes - Quantum Theory for the Perplexed, Wiley-VCH Verlag, Weinheim, Germany, 2005, Chapter 11.

20. S.D. Bartlett, T. Rudolph, R.W. Spekkens, Rev. Mod. Phys. 79, 555 (2007). 